Joel D. Velasco

Stanford University

\title{
When monophyly is not enough: \\ Exclusivity as the key to defining a phylogenetic species concept
}

abstract: A natural starting place for developing a phylogenetic species concept is to examine monophyletic groups of organisms. Proponents of "the" Phylogenetic Species Concept fall into one of two camps. The first camp denies that species even could be monophyletic and groups organisms using character traits. The second groups organisms using common ancestry and requires that species must be monophyletic. I argue that neither view is entirely correct. While monophyletic groups of organisms exist, they should not be equated with species. Instead, species must meet the more restrictive criterion of being genealogically exclusive groups where the members are more closely related to each other than to anything outside the group. I carefully spell out different versions of what this might mean and arrive at a working definition of exclusivity that forms groups that can function within phylogenetic theory. I conclude by arguing that while a phylogenetic species concept must use exclusivity as a grouping criterion, a variety of ranking criteria are consistent with the requirement that species can be placed on phylogenetic trees. 


\section{Introduction}

The species problem - how to sort organisms into various species remains a central problem in biological taxonomy. Despite many bitter disagreements about these fundamental units, there is widespread agreement on how to delimit "higher" taxa (those that are more inclusive than species). Systematics today is dominated by the phylogenetic perspective - the view that evolutionary history is of primary importance when delimiting taxa. From this standpoint, only monophyletic groups, which consist of an ancestor and all of its descendants, are taxa.

Phylogenetic systematics, or simply "phylogenetics", studies the diversity of life by considering the evolutionary history of various groups. It has been widely argued that a variety of species concepts in the literature recognize as species certain groups of organisms that are inappropriate for phylogenetic analysis (Rosen 1978, Eldredge and Cracraft 1980, Wiley 1981). For example, grouping organisms by their ability to interbreed, as the Biological Species Concept does, or by their shared ecological properties, as the Ecological Species Concept does, leads to groups that cannot be the tips of phylogenetic trees (Velasco 2008). If a group can be (properly) placed on a phylogenetic tree then the group in question can be subjected to phylogenetic analysis because it has a unique evolutionary history of the right sort. This unique history is what biological and ecological (and many other) "species" may lack.

Many authors have argued that while phylogenetics is an important biological project, other projects are important as well and can lead to different, 
but equally valid, ways of grouping organisms into species (Kitcher 1984, Ereshefsky 1992, Dupré 1993, Stanford 1995, LaPorte 2005). Authors with this view have defended a variety of forms of pluralism about species. Opposed to pluralism is monism which says that we need a single species concept so that the species taxa used for scientific communication throughout biology have one consistent meaning.

Among monists, a common viewpoint is that since phylogenetics is an overriding concern in taxonomy, any species concept which is inconsistent with phylogenetics is unacceptable. For the purposes of this paper, I adopt this phylogenetic perspective without argument. My question here is not whether we need a species concept that fits well within phylogenetics, but rather, what must such a phylogenetic species concept look like. My answer is that acceptable versions of a phylogenetic species concept must group organisms into genealogically exclusive groups. Roughly, these groups are such that each organism in the group is more closely related to any others in the group than to any organisms outside the group. Any concept that requires species to be exclusive groups, and only these concepts, can be properly called "phylogenetic" species concepts.

The paper proceeds as though we are aiming to develop the single best species concept. If it turns out that pluralism is in fact the best view, no such concept exists; however, the following discussion is still important because even then we will still need to know what the best species concept is for the purposes of phylogenetics. Determining necessary conditions for serving as tips of 
phylogenetic trees is an important project independently of whether monism or pluralism is true.

\section{Phylogenetic Species Concepts in the literature}

Phylogenetics examines the genealogical relationships among monophyletic groups in order to better understand their evolution through time. Serious problems arise when we attempt to investigate the history of nonmonophyletic groups using phylogenetic trees (Hennig 1966) and related problems occur with many species concepts (Velasco 2008). Therefore, we need a species concept that delimits groups based on common genealogical history if we are to form groups that fit into contemporary phylogenetic theory.

Authors who take such a genealogical perspective tend to develop some version of what they call a "Phylogenetic Species Concept" (PSC). Cracraft (1983) was perhaps the first to use this term. His version of the concept groups species by unique patterns of shared characters. Rosen (1978), Eldredge \& Cracraft (1980) and Nixon \& Wheeler (1990) each presented versions of the same basic idea. A more recent explication of this concept defines species as "the smallest aggregation of (sexual) populations or (asexual) lineages diagnosable by a unique combination of character states” (Wheeler and Platnick 2000: 58). This “diagnostic criterion” is not based only on evolutionary history, but rather, is based on character traits (genetic or phenotypic) and so can lead to nonmonophyletic groups forming species (Baum 1992, Baum and Donoghue, 1995). Thus, despite its name, this concept does not fit with phylogenetic theory. 
A different strain of phylogenetic species concepts has been produced with the explicit hope of co-opting the PSC name. Mishler \& Donoghue (1982), Donoghue (1985), Mishler (1985), Mishler \& Brandon (1987), and Mishler and Theriot (2000), each present versions of a PSC that allows only monophyletic groups to be species. These versions can often lead to different groupings than the "diagnostic" version of the PSC. For example, Mishler and Theriot (2000: 46, 47), takes its cue from Mishler and Brandon (1987) and says,

A species is the least inclusive taxon recognized in a formal phylogenetic classification. As with all hierarchical levels of taxa in such a classification, organisms are grouped into species because of evidence of monophyly.

They follow this with an odd description of how they are understanding monophyly, but this is not relevant here. What is important is that the definition of species (and all taxa) given by Mishler, Brandon, and Theriot explicitly builds in epistemological factors. On this picture, groups are not taxa because they are monophyletic; rather, they are taxa because there is strong evidence that they are monophyletic. The same considerations apply to species. Here, it seems that the authors are concerned about why we decide to name certain groups as species but naming a group as a species doesn't make it so. Unlike many forms of biological classification, a core idea in phylogenetics is that we do not create taxa, we discover them. These taxa are the products of the evolutionary process and exist independently of whether we ever correctly identify them or name them. The same considerations apply to species - when we name a group of organisms 
as a species, we are simply making a hypothesis that this group is in fact a species. It is possible that we are simply mistaken.

As we saw earlier, characters serve as evidence that a group is a taxon; they do not determine that it is a taxon. This is merely an instance of the general fact that taxa are not determined by any epistemological factors, but rather by objective, mind-independent facts about genealogical relationships. Given what Mishler and Theriot say elsewhere, it is clear that they do not intend their definition to rely on epistemological factors, and making this clear in the definition requires only a small wording change, but this change represents an important shift in the way that we view taxa and is therefore worth emphasizing.

Roughly speaking, we can think of these history-based versions of the PSC as requiring that species be monophyletic groups and then adding something about a ranking criterion. Because other kinds of taxa are monophyletic, we need a criterion to determine which monophyletic groups are species (as opposed to subspecies or genera etc.). These versions of the PSC (perhaps slightly tweaked) can be justified as follows: phylogenetic systematics requires that taxa be monophyletic; species are taxa; so species must be monophyletic.

Systematists tend to have one of two types of opinions about the above argument. The first type of response relies on the claim that species are fundamentally different kinds of entities than higher taxa. Higher taxa must be monophyletic, but because species play a different role in evolutionary theory than do other taxa, they are not (necessarily) monophyletic. Typically, these authors think that not only are species not required to be monophyletic, but that 
they couldn't be. It is a kind of category mistake to claim that they could be. The second response, which isn't critical, relies on thinking that species are not fundamentally different and so takes accepts the argument above at face value.

Neither of these views is entirely correct. It is a mistake to think that species are fundamentally different types of entities than higher taxa. When we are working within the confines of phylogenetic theory, species, like other taxa, must be genealogical units with certain properties. However, it is also incorrect to assume that there is a straightforward argument starting with higher taxa as monophyletic groups of species and transitioning to species being monophyletic groups of organisms. The relevant concepts can apply - but we will be grouping organisms incorrectly into species if we blindly apply the same principles in the case of species as are traditionally applied to higher taxa. We need to group organisms genealogically, but this is not simple. As it turns out, many monophyletic groups of organisms are not basic phylogenetic units. To see why this is the case, I will now discuss the two responses to the above argument in more detail.

\section{The argument that species can't be monophyletic}

The arguments from systematists who think that species cannot be monophyletic ultimately fail, but they nonetheless point us in the right direction for thinking about why it is none-trivial to apply the concept of monophyly to phylogenetic species. One common reason given for why species shouldn't be required to be monophyletic is that by definition, it is actually impossible for them 
to be so. After all, "A monophyletic group is a group of species descended from a single ('stem') species, and which includes all species descended from this stem species" (Hennig 1966:73). The idea is that terms like "monophyletic" and "paraphyletic" apply only to groups of "atoms" (the species) and not to the atoms themselves (Wiley 1981, Nixon \& Wheeler 1990).

However, even if we insist on this definition of monophyly, it is clear from context that this way of phrasing the objection to the requirement that species be monophyletic is merely semantic. The relevant question is whether we are required to group all of the descendants of some common ancestor together when we delimit taxa of any size or whether there can be exceptions at the species level. This is a question that all systematists must face regardless of how they wish to use the terms "monophyly" and "paraphyly" (de Queiroz and Donoghue 1990). For the purposes of clarity, I will continue to use the broader understanding of monophyly which allows that there are monophyletic groups of organisms and ask whether or not species should be required to be monophyletic.

Similarly, a second strain of "anti-species monophyly" claims that species can't be monophyletic because biparental organisms form reticulating (nonnested) patterns of genealogical relationship and the same holds of character distributions within such groups. Within such groups we find tokogeny, ancestordescendant relations, rather than phylogeny, sister-group relations (Hennig 1966, Meier and Willman 2000). Again, this is merely a semantic issue. Monophyletic groups of organisms still exist even if we deny that they can have internal phylogenetic relationships. 
What is underlying the distinction between species and higher taxa in many of these cases is not merely insistence on specific word usage, but rather, issues about epistemology. For example, de Queiroz and Donoghue deny that species can be monophyletic because "Phylogenetic methods break down in this case [the non-nested case] because an assumption underlying the principle that shared, derived characters provide evidence of phylogenetic relationship (i.e. of monophyly) is violated" (de Queiroz and Donoghue 1988: 325).

Nelson (1979) and Wheeler and Platnick (2000) discuss the failure of "cladistic methods" - by which they mean Parsimony - in such cases. Parsimony uses synapomorphies (shared derived traits) to judge relationships and assumes a tree-like branching structure. Many of these authors rely on the idea that the phylogeny is nothing but the results of applying a particular method to summarize character data. This is yet another instance of mistaking epistemology for metaphysics. Which organisms form genealogical groups and where these groups fit on the Tree of Life are objective facts that are metaphysically prior to any facts about how we might infer such relationships. A truly phylogenetic species concept relies on just these objective facts.

\section{The real problem with non-nested groups}

Although the fact that non-nested genealogical relationships might require different methods of inference than nested relationships require is no reason to doubt that concepts such as monophyly apply to groups of organisms, the issues raised above might be the beginnings of a more serious argument about why 
monophyletic groups of organisms may be unsuitable as species or as taxa more generally.

Part of what makes monophyletic groups so important in systematics is that they often form exclusive groups: groups in which all members in the group are more closely related to each other than to anything outside the group. Contemporary mammals form an exclusive group since any mammal is more closely related to any other mammal than to any non-mammal. In a purely diverging genealogy ${ }^{1}$, exclusive groups are formed by any time-slice of any monophyletic group as in Figure 1.

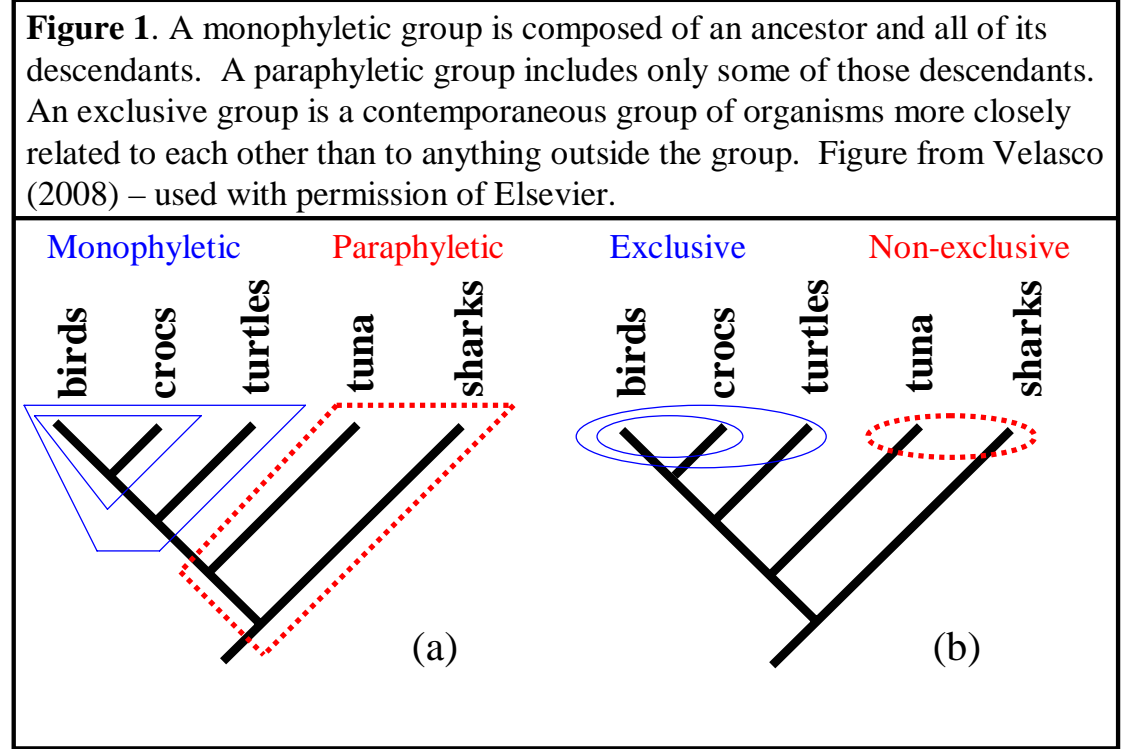

Here the concepts fit together nicely. In fact, Hennig (1966) assumed that monophyletic groups simply were exclusive groups and used the word "monophyletic" to refer to both properties. Immediately after defining 
monophyletic as a stem species plus all its descendants, he says "Another definition is: A monophyletic group is a group of species in which every species is more closely related to every other species than to any species that is classified outside the group" (Hennig 1966:73). It wasn't until de Queiroz and Donoghue (1990) that they were properly separated and the term "exclusivity" was invented and distinguished from monophyly. In addition, as with monophyly, the concept was extended to apply to groups of organisms generally and not just species (and the reliance on classification was removed to make it purely about genealogy). This "alternative definition" of monophyly partly explains why there is such confusion over whether there could be monophyletic groups of organisms. Such groups could strictly fit a more inclusive version of one of the definitions of monophyly, but they don't have all of the properties that monophyletic groups are supposed to have because they don't fit the other definition - i.e., they are not necessarily exclusive groups.

Once we distinguish monophyly from exclusivity, the conceptual issues become much easier to deal with. When we look at the appropriate level, the genealogies of organisms are not purely diverging. Many organisms have two parents, meaning that their genealogies will be reticulate. There will therefore be a separation between those groups that are monophyletic and those that are exclusive. The problem was there before with higher taxa (for example, when taxa are formed by hybridization), but it was typically ignored as being rare or unimportant to the general, overall pattern. But looking at groups of organisms 
directly brings the relevant issue to light in a way that makes it more easily appreciable.

A purely diverging phylogeny forms a tree - branches split off from each other but never merge. This will be the case if species always have exactly one immediate ancestor. In this respect, uniparental organisms form a genealogical tree in exactly the same way that taxa do. However, biparental organisms do not form a tree. This means that monophyletic groups will overlap and therefore will not form exclusive groups. For example, I am part of one monophyletic group stemming from my paternal grandfather consisting of him and all of his descendants. I am also part of another monophyletic group consisting of my maternal grandfather and all of his descendents. These groups overlap (my two sisters and I are part of both) and neither group is exclusive. We can see this by examining Figure 2, which depicts part of a rather ordinary human family tree.

Figure 2. Two monophyletic groups of organisms which overlap. Each consists of one grandparent, her children, and their children. No subgroup of organisms in the entire picture is exclusive (following the definition given in section 5).

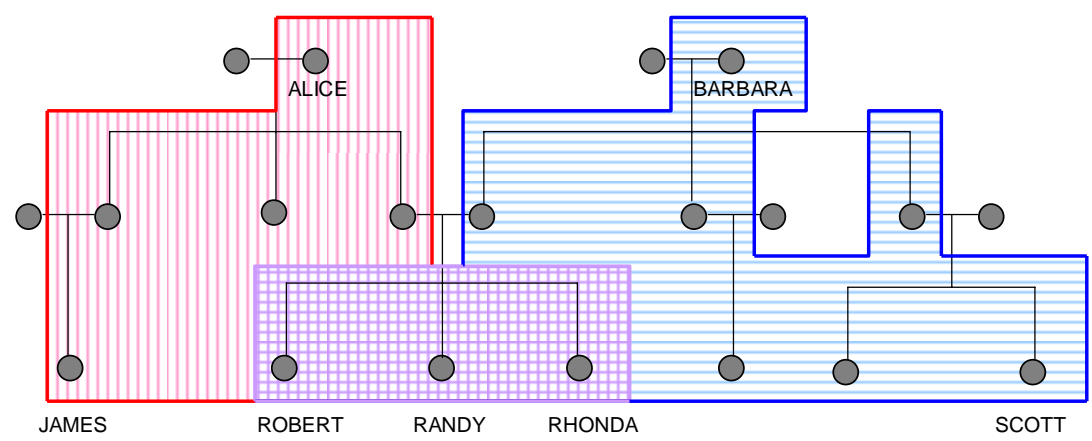


In this figure above, the two enclosed groups each represent monophyletic groups, but the organisms within each monophyletic group at the bottom (in the same generation) do not form exclusive groups. For example, James and Robert are first cousins since they share a grandparent. But Robert is also a cousin to Scott who is outside the left hand group.

Monophyletic groups of organisms are still interesting groups even if they overlap and are not exclusive- for example, if we are interested in tracking the history of some genetic trait, we may want to know all of the descendants of some particular person. A common example is tracking the recessive gene for hemophilia in the European royal families which requires us to have a pedigree containing at least Queen Victoria and all of her descendants. Not all of her descendants carry the relevant gene, but this would be the group to examine if you were interested in tracking it. The fact that this group overlaps with other monophyletic groups is irrelevant.

While biologically interesting groups can of course overlap, taxa cannot. Each organism is a member of at most one species and that species has a specific place in the nested taxonomic hierarchy. The idea of a single, unique taxonomy of life is the tradition within systematics and is enforced within various nomenclature codes such as the International Code of Botanical Nomenclature (McNeill et al. 2006). While it may be permissible to buck tradition and to violate various nomenclature codes, there is an additional problem with overlapping taxa. By allowing overlapping taxa, we will no longer be able to use species as the tips of phylogenetic trees, which is to deny our working premise 
that we are searching for a phylogenetic species concept. Again, even if we accept some version of pluralism which allows that species can overlap with each other, we would still need to know which groups can serve as "taxa" in the sense of being the tips of phylogenetic trees. These taxa cannot overlap. Since monophyletic groups do overlap, we can't simply allow all monophyletic groups to be taxa. However, exclusive groups do form a strict nested hierarchy and cannot overlap.

The fact that exclusive groups do not overlap is not simply due to wishful thinking but is a conceptual truth. To be clear, by non-overlap, I mean that if an organism is part of two exclusive groups $\mathrm{X}$ and $\mathrm{Y}$, either $\mathrm{X}$ is completely contained in $\mathrm{Y}$ or $\mathrm{Y}$ is completely contained in $\mathrm{X}$. Imagine two exclusive groups did overlap. Then there would be three organisms $a, b$, and $c$ where $a$ is in X, but not $\mathrm{Y}, b$ is in $\mathrm{X}$ and $\mathrm{Y}$, and $c$ is in $\mathrm{Y}$ but not $\mathrm{X}$. Now since $\mathrm{X}$ is exclusive, $b$ has to be closer to $a$ (which is in $\mathrm{X}$ ) than to $c$ (not in $\mathrm{X}$ ). Since $\mathrm{Y}$ is exclusive, $b$ has to be closer to $c$ (which is in $\mathrm{Y}$ ) than to $a$ (not in $\mathrm{Y}$ ). This is contradictory, so there can be no such overlap. Notice that this has nothing to do with X or Y being basal groups so higher taxa exhibit the same non-overlap property and therefore exclusive groups form a strict hierarchy which is a very desirable, if not necessary, condition on a definition of taxa.

Exclusive groups form a strict nested hierarchy and they are defined only in terms of genealogical history. Monophyletic groups meet the second condition, but not the first. We need shift the discussion of phylogenetic species from monophyletic groups to exclusive groups. 


\section{From monophyly to exclusivity}

Before we can safely define taxa as exclusive groups, we need to examine the properties of exclusive groups. To do this, we need a better understanding of what such groups look like in reticulating networks. While it seems as though I have already defined exclusivity - "each member is more closely related to anything else in the group than to anything outside it” - Hennig was thinking of groups of species in strictly diverging trees and it is not at all clear exactly how to think more generally of exclusive groups of organisms.

For example, on a natural understanding of "more closely related”, an organism is at least as closely related to one of its parents as to any other organism. But this would require that any exclusive group that contains me must also have my father and therefore all of his children. Since my father is in the group, so is his father and therefore all of my grandfather's children and of course their children as well, etc. Parallel reasoning leads to all of life forming one big exclusive group with no exclusive groups inside it. To avoid this consequence, we talk only about contemporaneous groups or relatively contemporaneous groups - perhaps we can include all living humans - but the point is that some ancestors must be excluded from the relevant comparison classes. This is what Baum and Shaw (1995) define as a "time-limited" rather than a "time-extended" approach to taxa. Baum (1998) describes how we could start with the timelimited approach to taxa and attempt to construct time-extended entities out of them. But as Baum points out, under almost any definition of species, we will 
need to be able to delimit a time-limited species in order to construct a timeextended species out of it, so there is nothing particularly surprising or undesirable about being forced into a time-limited approach. It is likely that Mishler and Brandon (1987) anticipated this issue without making the distinction between monophyletic and exclusive groups which is why they defined monophyly in a synchronic (or time-limited) way.

Incidentally, the time-limited approach to classification presents us with part of the solution to an apparent problem with phylogenetic species which we failed to deal with in section three. As Sober (2000: 166) points out, one species cannot be ancestral to another if that ancestral species were required to be monophyletic. Following Hennig (1966: 72), many systematists argue that classification is done from the standpoint of a particular "time horizon", but it isn't clear that this allows ancestral organisms to be a part of any species at all if all species are required to be monophyletic. On the time-limited view, the "ancestral species" can be exclusive (though not monophyletic) since it is being compared to its contemporaries and not its descendents.

Getting back to defining exclusivity, how do we measure how closely organisms are related to each other? An obvious way to do it is to measure their recency of common ancestry. A is more closely related to B than to C if the most recent common ancestor (MRCA) of A and B (or 'an' MRCA as there could be more than one) is more recent than an MRCA of A and C. ${ }^{2}$ This definition is applicable in both reticulating and diverging cases. However, we do have to be a bit more careful here since in a reticulating network, certain symmetries disappear 
- for example, unlike on a divergent tree, just because A is closer to B than to C is does not follow that B is closer to A than to C. For example, in the earlier Figure 2, while James is closer to Robert than to Scott, it is not true that Robert is closer to James than to Scott. As we will see, this asymmetry will prove the key to understand which properties of exclusivity are essential to being phylogenetic units.

The question now is how to extend this to some arbitrary group of organisms, A, contained within some reference group X (think of all contemporaneous organisms as the group $\mathrm{X}$ for the 'standard' example). There are a number of possibilities. One straightforward extension relies on pair-wise comparisons. For some subgroup A of $\mathrm{X}$ to be exclusive (relative to the rest of $\mathrm{X}$ ) is for any organism in A to be more closely related to any other organism in A than to any organism in $\mathrm{X}$ that is not in A. In this case, the relevant common ancestor of each pair might be different. For reasons that will become clear later, we often need to focus on groups that can be defined by talking about an MRCA of the entire group. A natural way to use exclusivity here would be to define an exclusive group as a group $\mathrm{A}$ in $\mathrm{X}$ such that $\mathrm{B}$, an MRCA of every member of $\mathrm{A}$, lived more recently than an MRCA of anyone in group A with anyone in X but not in A.

Both of these explications of exclusivity fail for the same reason: the group of organisms may not be a single, genealogical unit. Organisms inside the group (or ancestral organisms like an MRCA of the group) cannot represent the whole group on a phylogenetic tree. Organisms in different groups could still be 
connected to each other through lines of descent other than through this MRCA. This means that although group A would be exclusive, some organisms outside A could be closer to some organisms in A than to others. This is undesirable for a variety of reasons (Velasco 2008). As pointed out before, this possibility is due to the non-symmetry of 'more closely related than' in reticulating networks. Although group A would fit the definition of exclusivity, it doesn’t have all of the nice properties typically ascribed to exclusive groups (which we can now see should have been ascribed only to exclusive groups in a purely diverging phylogeny). A true genealogical unit should be such that it is "collapsible" and can be treated like a single point with respect to every other part of the Tree of Life: one organism in a group should represent the whole group (for purposes of taxonomy). To secure this, we want any contemporaneous organism outside a taxon to be equally closely related to everything inside that taxon. I will call this the unity condition.

This is one of the central reasons that monophyly is so important among the higher taxa. A time-slice of a paraphyletic group will always fail to meet the unity condition. It makes no sense to ask how closely related birds are to reptiles - it depends on which reptile you pick. This is not the case with mammals - with respect to birds, any mammal group is interchangeable.

Without this condition, intuitive consequences of genealogical relatedness claims will fail. Above the species level, when we say that birds and crocodiles are more closely related to each other than either is to snakes, we want this to imply that any particular species of bird will be closer to any crocodile species 
than to any species of snake. Similarly, below the species level, when we say that humans are closer to chimps than to gorillas, this should imply that any particular human is closer to any chimp than to any gorilla. If species do not meet the unity condition, this implication fails. If we want taxa to have a unique position on the Tree of Life and we want to be able to speak of the genealogy of a taxa or the phylogeny of a group of taxa, then taxa must meet the unity condition. We must understand exclusivity in a way that guarantees this.

In order to ensure that exclusive groups meet the unity condition, we must require that the MRCA of the group must be a part of any genealogical line leading to the exclusive group in question. One way to do this is to demand that the MRCA of the group be a "bottleneck" organism such that any ancestral line that reaches anything in A must go through this organism. But this is far too restrictive. Exclusive groups of this type would rarely form in sexually reproducing lineages. But we can ensure the unity condition without resorting to bottlenecks by simply requiring that there be some path through the MRCA. We can do this as follows: "An exclusive group of organisms $\mathrm{A}$ is a subgroup taken from a larger contemporaneous group $\mathrm{X}$ such that there is some organism $\mathrm{B}$ where B is an ancestor of every organism in A and B is a descendent of any common ancestor of any member of A and any member of X not in A.”

Notice that there is no need to require that the relevant organism B actually be the MRCA of all of the organisms in A. We still get the unity condition and everything else we want if there is some other common ancestor slightly further back in time that meets the other definitional criteria. It is also 
important to see that since B is an ancestor of every organism in A, A must include every contemporaneous descendant of B (any descendent of A would be equally closely related). So in addition to being exclusive, these groups are timeslices of monophyletic groups of organisms. But importantly, not all timeslices of monophyletic groups are exclusive so a synchronic understanding of monophyly is simply not what we were after all along.

As an example, let's describe what it means for extant humans to be an exclusive group. Let's call contemporaneous organisms the set of all organisms alive today. For the living humans to form an exclusive group there must be some organism B such that B is an ancestor of all living humans and is a descendent of any common ancestor of any human and a non-human. Any chimp and any human share very many ancestors in common, the most recent of which probably lived around 6 million years ago. If there is an organism B that is a descendant of each of these ancestors, then all humans are more closely related to each other than to any non-human, and any non-human is equally related to every human. This would mean that the unity condition applies to humans, showing that humans are an exclusive group.

We have good reason to believe that humans are an exclusive group under just such a definition. For example, Mitochondrial Eve, estimated to have lived about 140,000 years ago, (Dawkins 2004) is a matrilineal ancestor of every living human (trace back mother to mother to mother, etc. and you will hit Mitochondrial Eve). Likewise, Y-chromosome Adam, the patrilineal ancestor of every human, is estimated to have lived about 60,000 years ago. Either one, or 
many other ancestral organisms, could serve as "organism B" in our definition. Incidentally, the MRCA of all living humans (through mixed male and female lines) is estimated to have lived much more recently - estimates vary from 8,000 to as little as 2,000 years ago (Chang 1999; Rohde et al. 2004; Rohde ms). This seems remarkably recent, but in fact compared to other comparable groups of organisms, it is relatively distant due to the geographical separation of large groups of humans from each other. In a randomly mating population of $\mathrm{N}$ individuals, the predicted time to the MRCA is heavily concentrated around $\log _{2} \mathrm{~N}$ generations (Chang 1999). So, if humans were randomly mating, the population of 6,000,000,000 contemporaneous humans would be expected to have a MRCA who lived approximately 32 generations ago, which is less than 1000 years ago. It is not difficult for exclusive groups of this type to form given a reasonable amount of time isolated from outside groups.

While it may seem that we have an overly complicated definition of an exclusive group, it turns out to be easy for such relations to arise. Two isolated lineages are virtually certain to form exclusive groups in a biologically reasonable amount of time $\left(\log _{2} \mathrm{~N}\right.$ generations for a randomly mating population, longer if there is strong assortative mating or geographic structure). Exclusive groups of organisms certainly seem to be genealogical units of an interesting sort. Their genealogical connections with each other are grounded in the genealogical connections of organisms. Since they are time-slices of monophyletic groups they can function in historical explanations of trait distribution and since they possess the unity condition they can be placed at the tips of phylogenetic trees in order to 
allow history-based inferences in evolutionary biology. In short, exclusive groups fulfill all of the desiderata of the units in phylogenetic systematics and so exclusive groups of organisms can and should be equated with taxa. These taxa have the property of being located at a precise place on the Tree of Life. Though there is still room for debate about precisely how to construct time-extended entities out of these time-limited entities (Baum 1998), and whether we even want to do this (Baum forthcoming) these exclusive groups of organisms have the properties that we need for the building blocks of taxonomy. Whatever else we want to require of species, they must be exclusive groups of organisms.

\section{Species as a Rank}

What I have argued so far is that a phylogenetic species concept must group using exclusivity. But which exclusive groups should be species? This is a ranking question, separate from the grouping questions addressed so far. For our purposes, it is unnecessary to answer this question. What makes a species concept acceptable as "phylogenetic" is that species be able to serve at the tips of phylogenetic trees. This condition can be met with nearly any ranking criterion. Phylogenetics in general is consistent with a variety of views about the higher ranks such as order, family, and genus. It should come as no surprise that phylogenetic thinking about species shares this same quality. For example, Mishler and Brandon (1987) have a pluralistic view: different taxa are ranked as species for different reasons. Baum (forthcoming) agrees that a variety of ranking criteria can be used; however, he argues that it is "semi-arbitrary" which exclusive 
groups are species. This is a conventional matter to be decided by us. Mishler (1999) argues that we should get rid of the species rank all together. For Mishler, what is important is whether or not a group is a taxon. The "least-inclusive taxonomic unit" idea of Pleijel and Rouse (2000) is similar in spirit and content. These "anti-species" views are consistent with the above discussion, but do not follow by necessity. The species rank can be objective, semi-arbitrary, or even useless, but in each case, we could still talk of phylogenetic species as phylogenetic units. The debate about species ranking should be seen as incidental. Many species concepts, differentiated by their ranking criteria - or their view of the status of the species rank itself - have a right to be called "phylogenetic" species concepts. What unites them all is that they group organisms into exclusive groups.

\section{Endnotes}

1. By a 'diverging' phylogeny I mean that branches split but never join. Equivalently, each node (other than the root) has precisely one parent. It is tempting to think that a diverging phylogeny means that the horizontal distance between tips represents phenotypic divergence as well but this is not correct. For example, instances of convergent evolution take place on a diverging tree.

2. Baum and Shaw (1995) anticipate much of the above discussion and conclude that taxa should be exclusive groups, but deny that exclusivity can be understood 
in MRCA terms. They do this because they wish to define species as basal taxa and some exclusive groups would be too small to be species. However, here, I do not assume that species are the smallest exclusive groups; rather, I am searching for a general phylogenetic grouping criterion and a general understanding of exclusive groups of organisms.

\section{Acknowledgements}

Thanks to Matt Barker, David Baum, Marc Ereshefsky, Casey Helgeson, Brent Mishler, Greg Novack, Elliott Sober, Kim Sterelny, and John Wilkins, all of whom provided helpful comments on earlier drafts of this paper.

\section{References}

Baum, D. A. (1992). Phylogenetic species concepts. Trends in Ecology \& Evolution, 7(1), 1-2.

Baum, D. A. (1998). Individuality and the existence of species through time. Systematic Biology, 47(4), 641-653.

Baum, D.A. (forthcoming). Species as ranked exclusive groups. Systematic Biology.

Baum, D. A., \& Donoghue, M. J. (1995). Choosing among alternative "phylogenetic" species concepts. Systematic Botany, 20(4), 560-573. 
Baum, D. A. \& Shaw, K. L. (1995). Genealogical perspectives on the species problem. In P. C. Hoch \& A. G. Stephenson (Eds.), Experimental and Molecular Approaches to Plant Biosystematics (pp. 289-303). St. Louis, MO: Missouri Botanical Garden.

Chang, J. T. (1999). Recent common ancestors of all present-day individuals. Advances in Applied Probability, 31, 1002-1026.

Cracraft, J. (1983). Species concepts and speciation analysis. Current Ornithology, 1, 159-187.

Dawkins, R. (2004). The Ancestor's Tale. Houghton Mifflin Company, New York.

de Queiroz, K. \& Donoghue, M. (1988). Phylogenetic systematics and the species problem. Cladistics, 4, 317-338.

de Queiroz, K. \& Donoghue, M. (1990). Phylogenetic systematics or Nelson's version of Cladistics? Cladistics, 6, 61-75.

Donoghue, M. J. (1985). A critique of the biological species concept and recommendations for a phylogenetic alternative. The Bryologist, 88(3), $172-181$.

Dupré, J. (1993). The Disorder of Things: Metaphysical Foundations of the Disunity of Science. Harvard University Press, Cambridge, Massachusetts. 
Eldredge, N. \& Cracraft, J. (1980). Phylogenetic patterns and the evolutionary process : Method and theory in comparative biology. New York: Columbia University Press.

Ereshefsky, M. (1992). Eliminative pluralism, Philosophy of Science, 59, 671690.

Hennig, W. (1966). Phylogenetic Systematics. University of Chicago Press, Chicago.

Kitcher, P. (1984). Species. Philosophy of Science, 51, 308-333.

LaPorte, J. (2005). Is there a single objective, evolutionary tree of life? The Journal of Philosophy, 102(7), 357-374.

McNeill, J., Barrie, F. R., Burdet, H. M., Demoulin, V., Hawksworth, D. L., Marhold, K., Nicolson, D. H., Prado, J., Silva, P. C., Skog, J. E., Wiersema, J. H. \& Turland, N. J. (eds.) 2006. International Code of Botanical Nomenclature (Vienna Code) adopted by the Seventeenth International Botanical Congress Vienna, Austria, July 2005. Gantner Verlag, Ruggell, Liechtenstein.

Meier, R. \& Willman, R. (2000). The Hennigian species concept. In: Q.D. Wheeler and N. Platnick, eds. Species Concepts and Phylogenetic Theory, 44-54. New York: Columbia University Press. 
Mishler, B. D. (1985). The morphological, developmental, and phylogenetic basis of species concepts in bryophytes. The Bryologist, 88(3), 207-214.

Mishler, B. D., \& Brandon, R. N. (1987). Individuality, pluralism, and the phylogenetic species concept. Biology and Philosophy, 2, 397-414.

Mishler, B. D., \& Donoghue, M. J. (1982). Species concepts: A case for pluralism. Systematic Zoology, 31(4), 491-503.

Mishler, B. D. \& Theriot, E. C. (2000). The phylogenetic species concept (Sensu Mishler and Theriot). In: Q.D. Wheeler and N. Platnick, eds. Species Concepts and Phylogenetic Theory, 44-54. New York: Columbia University Press.

Nelson, G. (1979). Cladistic analysis and synthesis: Principles and definitions, with a historical note on Adanson's Familles des Plantes (1763-1764). Systematic Zoology, 28, 1-21.

Nixon, K., \& Wheeler, Q. (1990). An amplification of the phylogenetic species concept. Cladistics, 6(3), 211-223.

Pleijel, F. \& Rouse, G. W. (2000). Least-inclusive taxonomic unit: A new taxonomic concept for biology. Proceedings of the Royal Society of London Series B, 267, 627-630. 
Rohde, D.L.T., Olson, S., and Chang, J.T. (2004). Modelling the recent common ancestry of all living humans, Nature, 431(7008), 562-6.

Rohde, D.L.T. (submitted). On the common ancestors of all living humans. Submitted to American Journal of Physical Anthropology. Available online at: http://tedlab.mit.edu/ dr/

Rosen, D. E. (1978). Vicariant patterns and historical explanation in biogeography. Systematic Zoology, 27(2), 159-188.

Stanford, K. (1995). For pluralism and against realism about species. Philosophy of Science, 62, 70-91.

Velasco, J. D. (2008). Species Concepts Should Not Conflict with Evolutionary History, but often do. Studies in the History and Philosophy of Biological and Biomedical Sciences, 39, 407-414.

Wheeler, Q. D \& Platnick, N. (2000). The phylogenetic species concept (Sensu Wheeler and Platnick). In Q. D. Wheeler and N. Platnick (Eds). Species Concepts and Phylogenetic Theory (pp. 55-69). New York: Columbia University Press.

Wiley, E. (1981). Phylogenetics: The theory and practice of phylogenetic systematics. New York: Wiley-Interscience. 